\title{
Kinematical evidence for physically different classes of large-scale coronal EUV waves
}

\author{
A. Warmuth and G. Mann \\ Leibniz-Institut für Astrophysik Potsdam (AIP), An der Sternwarte 16, 14482 Potsdam, Germany \\ e-mail: awarmuth@aip.de
}

Received 9 February 2011 / Accepted 28 May 2011

\begin{abstract}
Context. Large-scale wavelike disturbances have been observed in the solar corona in the EUV range since more than a decade. The physical nature of these so-called "EIT waves" is still being debated controversially. The two main contenders are on the one hand MHD waves and/or shocks, and on the other hand magnetic reconfiguration in the framework of an expanding CME. There is a lot of observational evidence backing either one or the other scenario, and no single model has been able to reproduce all observational constraints, which are partly even contradictory. This suggests that there may actually exist different classes of coronal waves that are caused by distinct physical processes. Then, the problems in interpreting coronal waves would be mainly caused by mixing together different physical processes.

Aims. We search for evidence for physically different classes of large-scale coronal EUV waves.

Methods. Kinematics is the most important characteristic of any moving disturbance, hence we focus on this aspect of coronal waves. Identifying distinct event classes requires a large event sample, which is up to now only available from SOHO/EIT. We analyze the kinematics of a sample of 176 EIT waves. In order to check if the results are severely affected by the low cadence of EIT, we complement this with high-cadence data for 17 events from STEREO/EUVI. In particular, we focus on the wave speeds and their evolution.

Results. Based on their kinematical behavior, we find evidence for three distinct populations of coronal EUV waves: initially fast waves $\left(v \geq 320 \mathrm{~km} \mathrm{~s}^{-1}\right)$ that show pronounced deceleration (class 1 events), waves with moderate $\left(v \approx 170-320 \mathrm{~km} \mathrm{~s}^{-1}\right)$ and nearly constant speeds (class 2), and slow waves $\left(v \leq 130 \mathrm{~km} \mathrm{~s}^{-1}\right.$ ) showing a rather erratic behavior (class 3 ).

Conclusions. The kinematical behavior of the fast decelerating disturbances is consistent with nonlinear large-amplitude waves or shocks that propagate faster than the ambient fast-mode speed and subsequently slow down due to decreasing amplitude. The waves with moderate speeds are consistent with linear waves moving at the local fast-mode speed. Thus both populations can be explained in terms of the wave/shock model. The slow perturbations with erratic behavior, on the other hand, are not consistent with this scenario. These disturbances could well be due to magnetic reconfiguration.
\end{abstract}

Key words. Sun: activity - Sun: corona - Sun: flares - Sun: coronal mass ejections (CMEs) - waves - shock waves

\section{Introduction}

More than a decade has now passed since the Extreme ultraviolet Imaging Telescope (EIT; Delaboudinière et al. 1995) aboard the SOHO spacecraft first observed large-scale, globally propagating disturbances in the solar corona (Moses et al. 1997; Thompson et al. 1998). These perturbations have become known as "EIT waves", "coronal EUV waves", or simply coronal waves (for terminology, see Vršnak 2005). Despite intense scrutiny, the actual physical nature of these perturbations is still being debated very controversially (see the reviews by Warmuth 2007; Wills-Davey \& Attrill 2009; and Gallagher \& Long 2010).

There are basically two different models for coronal waves: in the wave/shock scenario, the perturbations are interpreted in terms of fast-mode MHD waves and/or shocks (see e.g. Warmuth et al. 2004b; Grechnev et al. 2008; Temmer et al. 2009). These waves could be either driven by a piston or freely propagating, and they could be launched and/or driven by flares, CMEs, or small-scale ejecta. While the main disturbance is commonly considered as a fast-mode wave due to its speed and the fact that it can propagate normal to the magnetic field lines, several authors have studied the contribution of slow-mode waves (see Wu et al. 2005; Gilbert et al. 2008; Wang et al. 2009; Podladchikova et al. 2010). A different kind of wave model - a slow-mode soliton - was proposed by Wills-Davey et al. (2007). In contrast, the magnetic reconfiguration scenario (e.g. Delannée \& Aulanier 1999; Delannée et al. 2008; Attrill et al. 2007; Dai et al. 2010) interprets the observed disturbances as the consequence of a magnetic restructuring of the corona in the framework of an expanding CME, which could generate both propagating and standing wavefronts (Delannée 2000). Finally, both scenarios can be combined: in numerical simulations of erupting flux ropes, propagating disturbances caused by both processes could be identified (Chen et al. 2002, 2005), and Zhukov \& Auchère (2004) found observational evidence for both a wave mode and an eruptive mode in EIT wave events.

Kinematics is the most basic characteristic of any moving disturbance, thus a lot of evidence for or against a certain model has been derived from kinematical studies. For the fast and strong waves, which might represent the large-amplitude limit of the phenomenon, ubiquitous deceleration was found (e.g. Warmuth et al. 2004a; Warmuth 2010). Deceleration also accounts for the apparent velocity discrepancy between Moreton 
and EIT waves: the low temporal cadence of EIT leads to an undersampling of the early phase of the wave's propagation where it is still fast, resulting in lower EIT wave speeds (Warmuth et al. 2001). An alternative explanation of this discrepancy is provided by the model of (Chen et al. 2002), where Moreton and EIT wave are interpreted as two separate physical entities. However, both the studies combining wave signatures from different instruments (e.g. Vršnak et al. 2002; Warmuth et al. 2004a, 2005; Grechnev et al. 2008) as well as recent observations with STEREO/EUVI (e.g. Long et al. 2008; Veronig et al. 2008; Gopalswamy et al. 2009) have consistently shown that - at least in the case of fast and strong waves - all wavefronts are consistent with a single decelerating disturbance. The initial speeds of more than $600 \mathrm{~km} \mathrm{~s}^{-1}$ imply that these disturbances are initially at least weakly shocked (e.g. Narukage et al. 2002; Hudson et al. 2003; Warmuth et al. 2005).

This scenario has been challenged by the fact that some EIT waves have speeds that are clearly below the coronal Alfvén and sound speeds (Wills-Davey et al. 2007), and by some of the new high-cadence EUV data available from STEREO/EUVI. Zhukov et al. (2009) have reported an EUVI wave showing a very erratic kinematical behavior, which strongly argues against a wave or shock nature of the disturbance. In other events, constant speeds were found (e.g. Patsourakos \& Vourlidas 2009; Ma et al. 2009; Veronig et al. 2010) that could imply small-amplitude (i.e., linear) waves. We thus have to stress that now waves with dissimilar kinematical behavior have been positively identified - insufficient cadence is no longer an explanation for any discrepancies. These different kinds of kinematical behavior are the the main reason for the still lacking consensus regarding the physical nature of coronal waves: no single model is capable of reproducing all different kinds of behavior.

However, are we actually sure that a single model is really appropriate for coronal waves? In other words, are we really sure that the phenomena we call "coronal waves" are all caused by the same physical process? What if there are several distinct processes which could generate wavelike phenomena in the corona? In that case, a large part of the controversy would result from confusing different types of events and trying to force them under a single physical model. In this context, it is interesting to note that the studies concentrating on fast and strong coronal waves - those associated with chromospheric Moreton waves (Moreton 1960) and metric type II radio bursts - predominantly find evidence backing the wave/shock scenario (e.g. Klassen et al. 2000; Narukage et al. 2002; Warmuth et al. 2004b; Vršnak et al. 2006; Grechnev et al. 2008; Asai et al. 2008; Gopalswamy et al. 2009), while those focusing on slower waves - or focusing solely on EIT data - tend to favor the magnetic reconfiguration scenario (e.g. Delannée 2000; Attrill et al. 2007; Zhukov et al. 2009; Dai et al. 2010), or slow-mode solitons (Wills-Davey et al. 2007).

We therefore have to search for evidence for the existence of physically different classes of coronal wave events. In this paper, we will focus on kinematics. Since identifying distinct event classes requires a large event sample, we use the comprehensive catalog of SOHO/EIT waves compiled by Thompson \& Myers (2009). We analyze the kinematics of this large event sample, focusing on the wave speeds and their evolution (Sect. 2). In order to check if the results are severely affected by the low cadence of EIT, we complement this with high-cadence data for 17 events from STEREO/EUVI (Sect. 3). In Sect. 4 we will discuss our results, and the conclusions will be given in Sect. 5 .
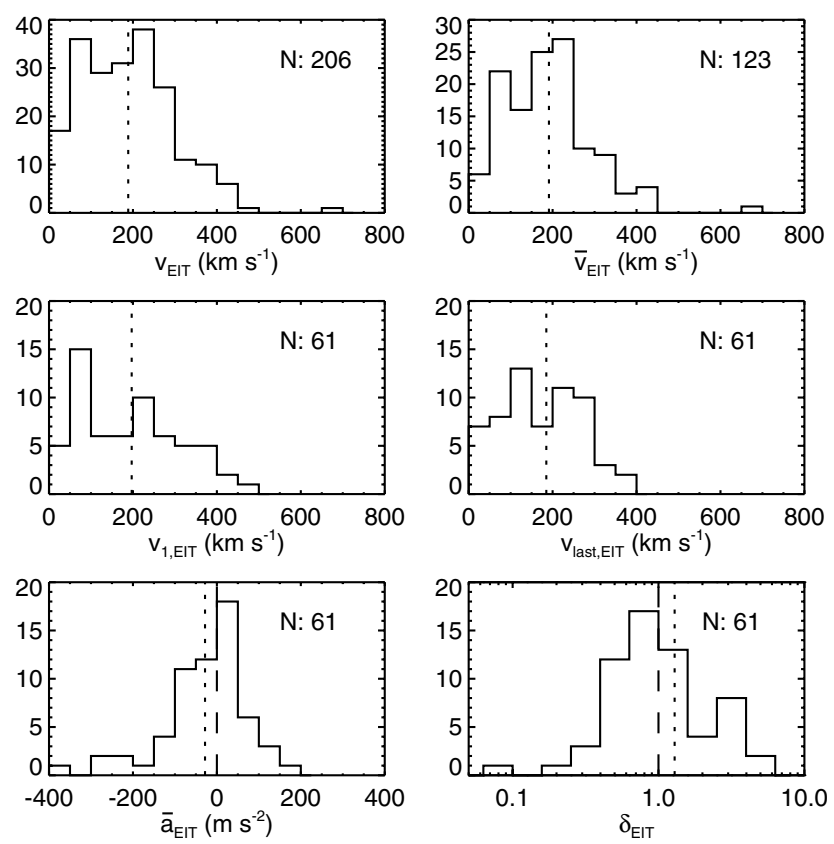

Fig. 1. Histograms of all measured EIT wave speeds $v_{\text {EIT }}$, the mean speeds $\bar{v}_{\text {EIT }}$, the initial speeds $v_{1, \text { EIT }}$, the final speeds $v_{\text {last,EIT }}$, the mean acceleration $\bar{a}_{\mathrm{EIT}}$, and the power-law index $\delta_{\mathrm{EIT}}$, as derived from the catalog of Thompson \& Myers (2009). The dotted vertical lines shows the mean values of the distributions, and $N$ indicates the total number of individual values contained in each distribution. The dashed vertical lines indicate constant speeds with $\bar{a}_{\text {EIT }}=0$ and $\delta_{\text {EIT }}=1$.

\section{EIT wave kinematics}

The EIT (Delaboudinière et al. 1995) aboard the SOHO provides full-disk EUV images of the solar corona with a spatial resolution of 2 ". $6 /$ pixel in four different passbands. Under the normal observing regime, only the Fe XII channel (195 ^, with a peak emission at $T \sim 1.5 \mathrm{MK}$ ) has sufficient cadence - typically $15 \mathrm{~min}$ - to observe more than one wavefront in a wave event. Thompson \& Myers (2009) have compiled a comprehensive catalog of 176 EIT waves, covering the time interval from January 1997 to June 1998. The catalog contains the measured speeds of the waves based on the leading edges of consecutive wavefronts that were determined visually. These speeds were measured both in the plane of sky and projected on the solar surface - we will use the latter method since it is more appropriate for coronal waves. The speeds were measured for a single direction representing the main propagation direction. A single speed (i.e., two successive wavefronts) was measured in 62 events, two speeds in 43 events, three speeds in 14 events, and four speeds in four events.

Figure 1 shows histograms for all EIT wave speeds and derived kinematical parameters, as measured by Thompson \& Myers (2009). Generally, the histograms show that although many EIT waves do fall within the commonly cited speed range (some 200-300 $\mathrm{km} \mathrm{s}^{-1}$ ), they have a rather broad distribution. The histogram of all measured wave speeds $v_{\text {EIT }}$ (top left) shows a wide range of wave velocities, from $15 \mathrm{~km} \mathrm{~s}^{-1}$ to $654 \mathrm{~km} \mathrm{~s}^{-1}$, with a mean of $\left\langle v_{\mathrm{EIT}}\right\rangle=189 \mathrm{~km} \mathrm{~s}^{-1}$. Note that the distribution is characterized by two distinct maxima, centered around 100 and $200 \mathrm{~km} \mathrm{~s}^{-1}$, as well as by an extended tail beyond $300 \mathrm{~km} \mathrm{~s}^{-1}$. Basically the same distribution is found for the mean speeds of the individual waves, $\bar{v}_{\text {EIT }}$ (top right). 
We now consider only the 61 waves which have been adequately resolved kinematically, which means that at least two speeds (three fronts) could be measured. The histogram of the

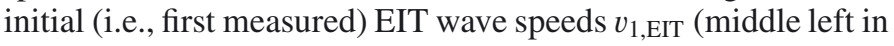
Fig. 1) shows a very pronounced separation between the peak at low and higher speeds, and the higher-speed peak is immediately followed by a high-speed tail. In comparison, the last measured speeds in the individual events, $v_{\text {last,EIT }}$ (middle right), still show a similar distribution, but with a less pronounced separation of the peaks and a less developed high-speed tail. The respective mean values of the distributions are $\left\langle v_{1, \mathrm{EIT}}\right\rangle=195 \mathrm{~km} \mathrm{~s}^{-1}$ and $\left\langle v_{\text {last,EIT }}\right\rangle=171 \mathrm{~km} \mathrm{~s}^{-1}$. These moderate differences imply that at least not all EIT waves are propagating at a constant speed.

To quantify the changes in speed during the waves' propagation, we use two methods that are well established in the study of coronal waves (e.g. Warmuth et al. 2001, 2004a; Grechnev et al. 2008; Narukage et al. 2008; Muhr et al. 2010). One is to fit the distances of the wavefronts from the center of origin by a 2 nd degree polynomial, which is equivalent to fitting the speeds with a linear function. This yields a constant acceleration, $\bar{a}$ (which, when negative, implies deceleration). On the other hand, we can fit the kinematical curve with a power-law of the form $d=c\left(t-t_{0}\right)^{\delta}$, where $d$ is the distance, $c$ is a constant, $t-t_{0}$ is the time relative to the initiation time of the wave, and $\delta$ is the power-law index (cf. Warmuth et al. 2004a). In contrast to the quadratic function, the power-law yields a time-dependent acceleration, namely $a(t)=c(\delta-1) \delta\left(t-t_{0}\right)^{\delta-2}$. The two procedures are frequently used since they are the simplest functions (three parameters each) that can reproduce a monotonically changing speed.

The resulting distribution for $\bar{a}_{\text {EIT }}$ is centered around zero (lower left in Fig. 1), showing that many waves are indeed consistent with nearly constant speeds. However, there is a small tail of higher accelerations (up to $\bar{a}_{\text {EIT }}=180 \mathrm{~m} \mathrm{~s}^{-2}$ ) and a much more pronounced tail of clearly decelerating waves (down to $\bar{a}_{\text {EIT }}=-370 \mathrm{~m} \mathrm{~s}^{-2}$ ). These decelerating waves shift the mean of the distribution to $\left\langle\bar{a}_{\mathrm{EIT}}\right\rangle=-28 \mathrm{~m} \mathrm{~s}^{-2}$. The power-law indices $\delta_{\text {EIT }}$ (lower right in Fig. 1) also have a rather broad distribution.

While the distribution of the speeds - especially the two distinct peaks - suggests that there may be more than one class of wave events, the situation is less clear-cut in the case of $\bar{a}_{\text {EIT }}$ and $\delta_{\text {EIT }}$. To investigate this matter in more detail, we have to look for relationships between the speeds and their evolution as parameterized by $\bar{a}_{\text {EIT }}$ and $\delta_{\text {EIT }}$. Of particular interest is the evolution of a wave with a given initial speed $v_{1}$ with time. Thus we have plotted the initial EIT wave speeds $v_{1, \text { EIT }}$ versus the mean EIT wave accelerations $\bar{a}_{\text {EIT }}$ in Fig. 2 (preliminary results of this kind of analysis have been reported by Warmuth 2010). Here, the error bars are based on assuming an error in the distance measurements of $\sigma_{d}=15 \mathrm{Mm}$, corresponding to about eight EIT pixels. While this overestimates the error for sharp wavefronts, it accounts for poorly defined events.

Inspecting Fig. 2, we note that the events are clustered into three different groups which had already appeared in the histograms of the speeds. Starting at the higher speeds, the highspeed tail of the velocity distributions can be identified as a cluster of 12 events with initial speeds $v_{1 \text {,EIT }} \geq 325 \mathrm{~km} \mathrm{~s}^{-1}$ which all show deceleration $\left(\bar{a}_{\text {EIT }}<0\right)$. In addition, there is a clear correlation between speeds and the amount of deceleration: the faster the wave is initially, the stronger is the deceleration. The mean of the deceleration is $\left\langle\bar{a}_{\mathrm{EIT}}\right\rangle=-151 \mathrm{~m} \mathrm{~s}^{-1}$ for this group of waves.

Below $v_{1 \text {,EIT }}=325 \mathrm{~km} \mathrm{~s}^{-1}$ we find a second cluster of points (23 events) extending down to $v_{1, \text { EIT }}=170 \mathrm{~km} \mathrm{~s}^{-1}$. No clear correlation between speed and acceleration is apparent here - both

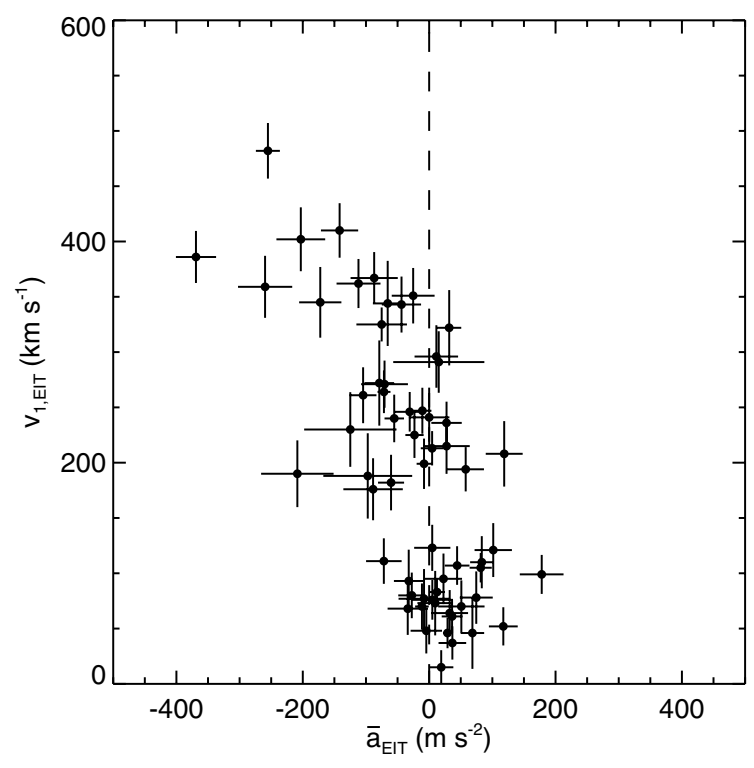

Fig. 2. Initial EIT wave speed, $v_{1, \text { EIT }}$, versus mean EIT wave acceleration, $\bar{a}_{\text {EIT }}$. The dashed vertical line indicates constant speed $\left(\bar{a}_{\text {EIT }}=0\right)$.

moderate levels of acceleration and deceleration are found, the mean value being $\left\langle\bar{a}_{\text {EIT }}\right\rangle=-32 \mathrm{~m} \mathrm{~s}^{-1}$. Given the error bars, the majority of these events are still consistent with nearly constant speeds.

Very clearly separated from this second group of events a third one is evident at speeds below $v_{1, \text { EIT }}=130 \mathrm{~km} \mathrm{~s}^{-1}$, comprising 26 events. Just like in the case of the second cluster, no correlation between speed and acceleration is evident here, both moderate acceleration and deceleration is present, with a mean value of $\left\langle\bar{a}_{\text {EIT }}\right\rangle=31 \mathrm{~m} \mathrm{~s}^{-1}$. The majority of these events show slight acceleration, but again many of them are consistent with nearly constant speeds.

Based on the relation between initial speed and mean acceleration we have thus identified waves with three different kinds of kinematical behavior. Does this result also hold up when we use power-laws instead of 2 nd degree polynomials? This is shown in Fig. 3, where the initial EIT wave speed $v_{1, \text { EIT }}$ is plotted versus the power-law index $\delta_{\text {EIT }}$. Indeed we can identify basically the same groups of events: fast and clearly decelerating waves $\left(\delta_{\text {EIT }}<1\right)$, waves with moderate speeds and only slight acceleration or deceleration, and well separated slow waves that mostly show slight acceleration.

The same three groups can be identified when the mean wave speed $\bar{v}_{\text {EIT }}$ is considered instead of the initial one, although the separation between the groups is less clear in that case. However, if the last measured speeds $v_{\text {last,EIT }}$ are used instead, only a single cluster of value pairs without evident correlations or subgroups is obtained. This implies that the initial wave speed is the most important factor which determines the kinematical evolution of the wave, while the final wave speed does not seem to be essential in this respect.

One caveat is necessary here: the low cadence of EIT can introduce various artifacts that may lead to incorrect conclusions. One possibility is that a wave which is initially very fast and decelerates strongly will be sampled not sufficiently in order to reflect that behavior. In Fig. 2, such an event will thus appear at a lower speed and a smaller amount of deceleration, i.e., it will be shifted from the upper left corner of the plot towards the center. Conversely, a wave with constant speed can be recorded 


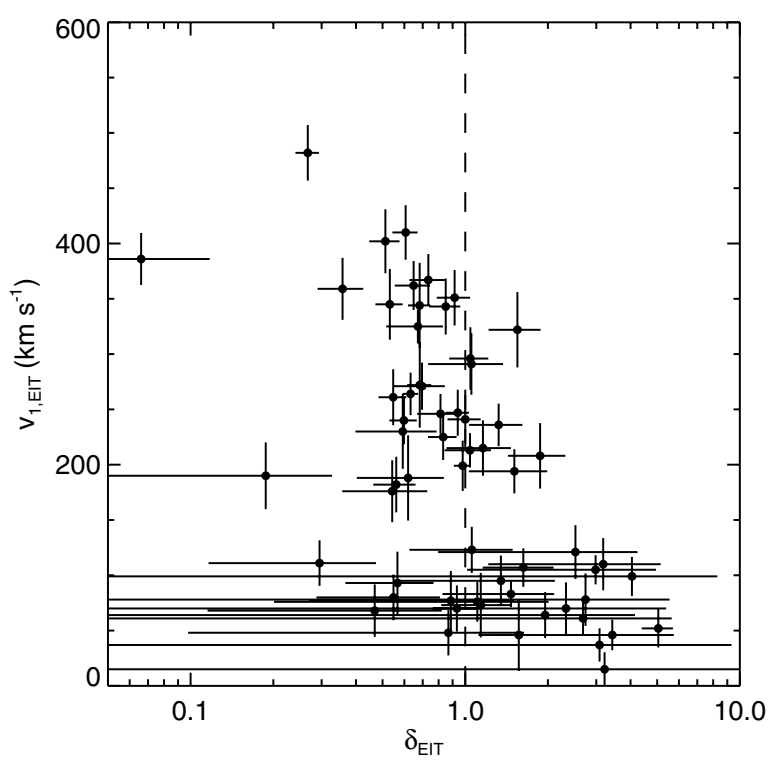

Fig. 3. Initial EIT wave speed, $v_{1, \text { EIT }}$, versus power-law index, $\delta_{\text {EIT }}$. The dashed vertical line indicates constant speed $\left(\delta_{\text {EIT }}=1\right)$.

as a strongly decelerating one if the wave is stopped at a coronal hole boundary (Thompson et al. 1998) or a separatrix between active regions (Delannée \& Aulanier 1999). A clear example of such a case can be seen as an outlier in Figs. 2 and 3 at $v_{1, \text { EIT }}=180 \mathrm{~km} \mathrm{~s}^{-1}, \bar{a}_{\text {EIT }}=-200 \mathrm{~m} \mathrm{~s}^{-2}$ and $\delta_{\text {EIT }}=0.2$. This is a wave which is decelerating much more strongly than other waves with moderate speeds. Visual inspection of the EIT images reveals that the wave indeed stops at a separatrix.

The low cadence of EIT can thus strongly modify kinematical plots, possibly leading to erroneous conclusions. Therefore, it is necessary to check these EIT-based results with wave observations that have a significantly better cadence.

\section{EUVI wave kinematics}

The Extreme UltraViolet Imager (EUVI; Wuelser et al. 2004) is part of the Sun Earth Connection Coronal and Heliospheric Investigation (SECCHI; Howard et al. 2008) instrument suite aboard the STEREO-A (ahead) and STEREO-B (behind) spacecraft and provides full-disk images with a spatial resolution of 1.'6/pixel. For this study, we used the Fe IX (171 $\AA, T \sim 1 \mathrm{MK})$ and Fe XII (195 $\AA, T \sim 1.5 \mathrm{MK})$ channels with cadences of 1.25-5 min and 5-10 min, respectively. Compared to EIT, the higher cadence results in a significantly improved resolution of the waves' kinematics: while the mean number of detected fronts per event is just 2.2 for EIT in the sample of Thompson \& Myers (2009), this number rises to 5.9 (EUVI $195 \AA$ A) and 8.1 (EUVI $171 \AA$ ) for the EUVI waves studied in the present work. This removes artifacts like the ones discussed above for EIT.

We have chosen 17 wave events observed by EUVI (an order of magnitude less than EIT waves), shown in Table 1. Apart from the kinematical parameters that will be discussed in the following, the table gives the time of the first wavefront visible in the $195 \AA$ channel, $t_{1,195}$, and the spacecraft (A or B) which was used in the present analysis (note that three of the earlier events were actually observed by both spacecraft). While all waves were observed in $195 \AA$ only nine had $171 \AA$ coverage (all events up to 2010 Jan. 17, and 2010 Sep. 08). Some of the events have already
Table 1. EUVI wave events.

\begin{tabular}{lccccc}
\hline \hline Date & $\begin{array}{c}t_{1,195} \\
(\mathrm{UT})\end{array}$ & $\mathrm{SC}$ & $\begin{array}{c}v_{1,195} \\
\left(\mathrm{~km} \mathrm{~s}^{-1}\right)\end{array}$ & $\begin{array}{c}\bar{a}_{195} \\
\left(\mathrm{~m} \mathrm{~s}^{-2}\right)\end{array}$ & $\delta_{195}$ \\
\hline 2007 May 19 & $12: 52$ & $\mathrm{~A}$ & 391 & -119 & 0.64 \\
2007 Dec. 07 & $04: 36$ & $\mathrm{~A}$ & 234 & 27 & 1.16 \\
2007 Dec. 08 & $17: 26$ & $\mathrm{~A}$ & 32 & 66 & 9.11 \\
2007 Dec. 31 & $00: 56$ & $\mathrm{~B}$ & 409 & -196 & 0.38 \\
2008 Apr. 26 & $13: 56$ & $\mathrm{~B}$ & 245 & 75 & 1.83 \\
2009 Feb. 13 & $05: 35$ & $\mathrm{~B}$ & 235 & -15 & 0.9 \\
2009 Dec. 22 & $04: 56$ & $\mathrm{~A}$ & 521 & -284 & 0.68 \\
2010 Jan. 17 & $03: 51$ & $\mathrm{~B}$ & 284 & -32 & 0.84 \\
2010 Apr. 28 & $22: 36$ & $\mathrm{~B}$ & 272 & -48 & 0.79 \\
2010 Apr. 29 & $00: 25$ & $\mathrm{~B}$ & 223 & -2 & 0.98 \\
2010 Apr. 29 & $02: 55$ & $\mathrm{~B}$ & 321 & -38 & 0.72 \\
2010 Apr. 29 & $06: 27$ & $\mathrm{~B}$ & 376 & -12 & 0.93 \\
2010 Jun. 12 & $00: 56$ & $\mathrm{~A}$ & 487 & -171 & 0.74 \\
2010 Jun. 13 & $05: 38$ & $\mathrm{~A}$ & 308 & 40 & 1.07 \\
2010 Aug. 07 & $18: 06$ & $\mathrm{~B}$ & 422 & -181 & 0.63 \\
2010 Aug. 14 & $09: 51$ & $\mathrm{~A}$ & 503 & -239 & 0.54 \\
2010 Sep. 08 & 23:18 & $\mathrm{A}$ & 377 & -233 & 0.6 \\
\hline
\end{tabular}

Notes. For details see main text.

been studied by other authors: 2007 May 19 (see Long et al. 2008; Veronig et al. 2008; Gopalswamy et al. 2009; Chen et al. 2010), 2007 Dec. 7 (Ma et al. 2009; Patsourakos et al. 2009), 2007 Dec. 8 (Zhukov et al. 2009), 2007 Dec. 31 (Dai et al. 2010), 2009 Feb. 19 (Patsourakos \& Vourlidas 2009; Kienreich et al. 2009; Cohen et al. 2009), 2010 Jan. 17 (Veronig et al. 2010), and the four waves of April 2010 (Kienreich et al. 2011).

We used running difference images to visually determine the leading edge of the wavefronts. Then the distance of the leading edge from the extrapolated wave radiant point was measured along 10 great circles (cf. Warmuth et al. 2004a) ${ }^{1}$. The distances were fitted with 2 nd degree polynomials and power-laws, as discussed in Sect. 2. Table 1 gives the initial wave speeds $v_{1,195}$ (derived from the two earliest wavefronts), mean accelerations $\bar{a}_{195}$, and power-law indices $\delta_{195}$, all derived from $195 \AA$ observations.

In particular, we are interested to see if the EUVI observations confirm the result that the waves show different kinematical behaviors depending to their initial speeds. As an example, Fig. 4 shows the distances $d$ (actually, $\left\langle d_{i}\right\rangle$ as averaged over the 10 individual paths is plotted, with the error bars given by the standard deviation of $d_{i}$ ) as well as the derived speeds $v$ as a function of time $t$ for two waves with high initial speeds (2007 May 19 and 2009 Dec. 22). The wavefront distances measured in the $195 \AA$ and $171 \AA$ images map out the same kinematical curves and are thus consistent with a single physical disturbance (cf. Long et al. 2008). It is clearly evident that in both events the waves are decelerating: they start off at $v_{1}=600-700 \mathrm{~km} \mathrm{~s}^{-1}$ and then decelerate to $v \approx 300 \mathrm{~km} \mathrm{~s}^{-1}$. The mean decelerations are in the range of a few $100 \mathrm{~m} \mathrm{~s}^{-2}$, and the power-law index is $\delta=0.57-0.67$. Note that the power-law, with a lower $\chi_{\text {red }}^{2}$ value, actually provides a better fit than the polynomial.

In contrast, initially slower EUV waves have a very different behavior, as shown for two examples (2007 Dec. 07 and

\footnotetext{
1 Note that we thus measure the projection of a $3 \mathrm{D}$ wavefront onto the solar surface, which could in principle result in different kinematics as compared to the true 3D geometry. However, Ma et al. (2009) report significant differences of the wavefronts imaged from the different perspectives of both STEREO spacecraft only for the early phase of a wave's evolution, which is of less relevance to the current study. We have verified this by measuring three of our events with STEREO A and $\mathrm{B}$, and finding consistent results.
} 
A. Warmuth and G. Mann: Kinematical evidence for physically different classes of large-scale coronal EUV waves
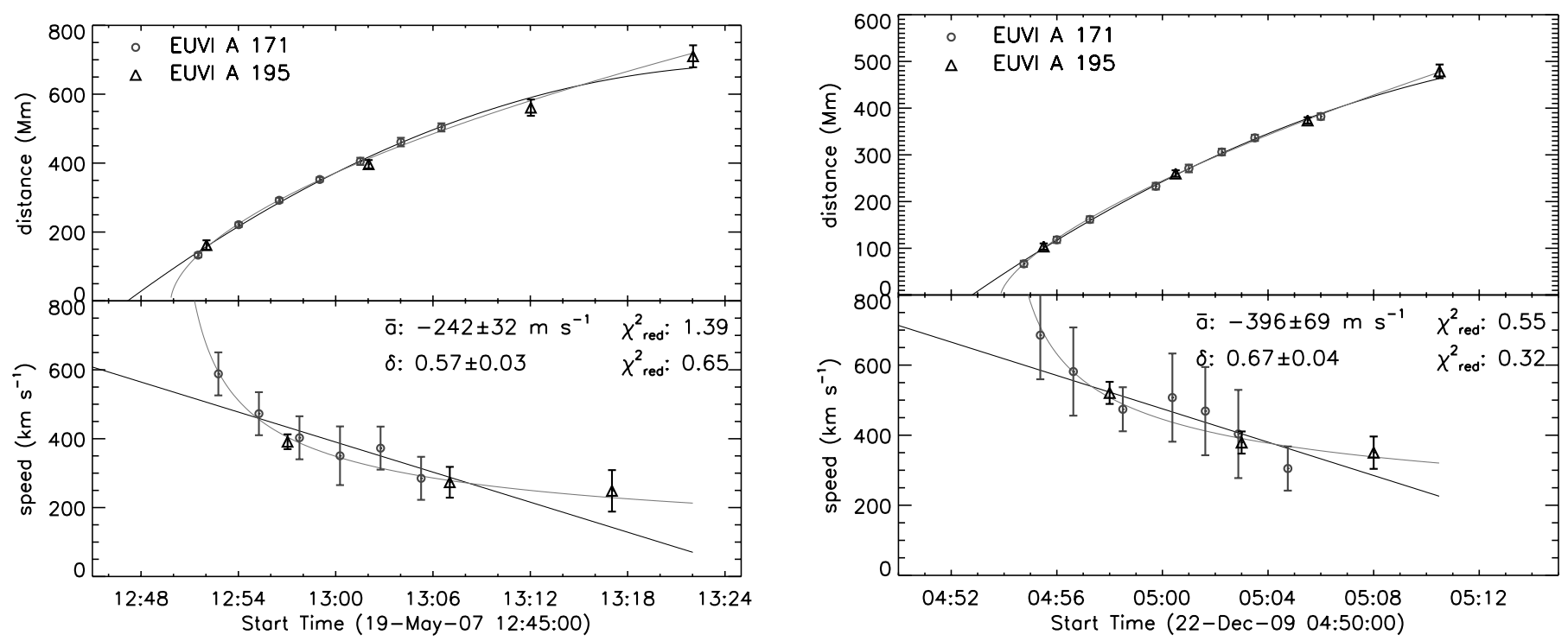

Fig. 4. Kinematics of two fast coronal waves observed with STEREO-A/EUVI (left: 2007 May 19; right: 2009 Dec. 22). The plots show the deprojected distance $d$ of the leading edges of the wavefronts from the extrapolated radiant point (upper half of the plots), and the corresponding speeds $v$ (lower half), both as a function of time $t$. Also shown are 2nd degree polynomial (black line) and power-law fits (grey line), and the corresponding mean acceleration $\bar{a}$ and the power-law index $\delta$ is indicated. To the right of $\bar{a}$ and $\delta$, the reduced chi-squared values $\chi_{\text {red }}^{2}$ for the polynomial and power-law fit is given, respectively. Note the pronounced deceleration of the waves, and the fact that the power-law provides a better fit than the polynomial.
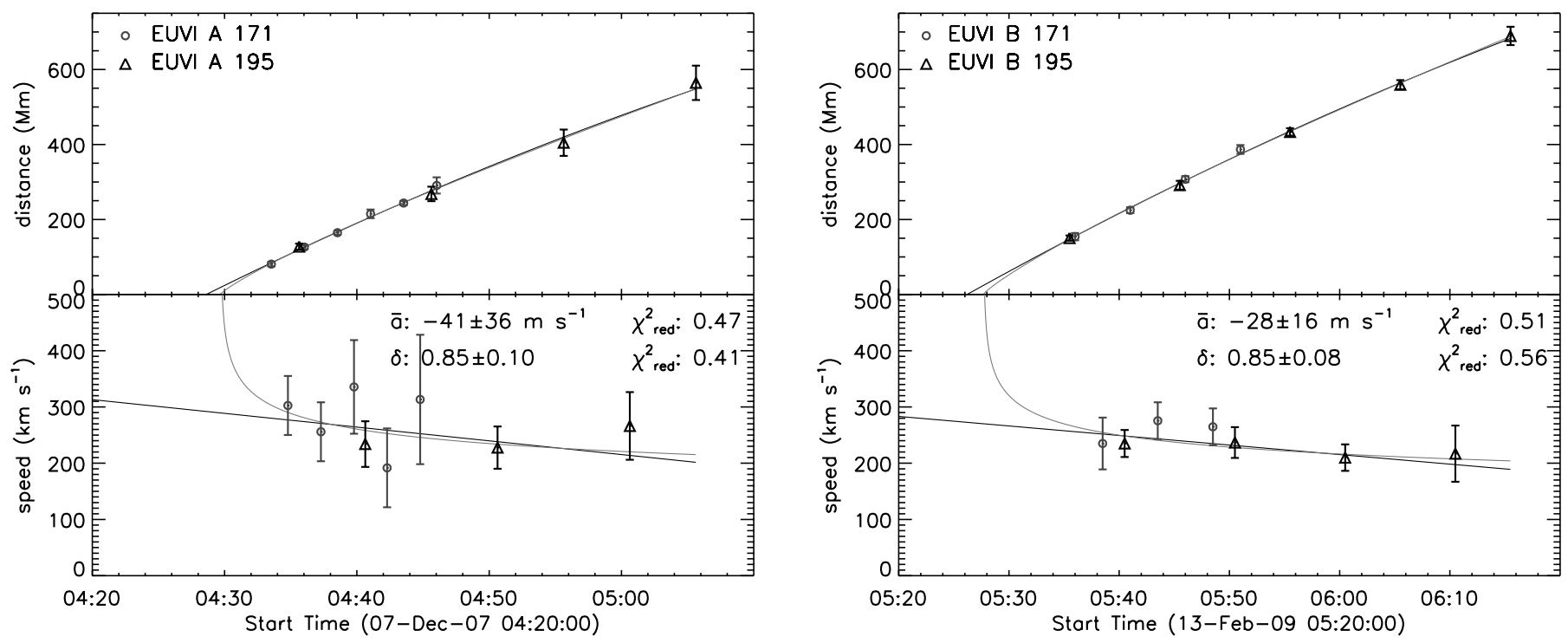

Fig. 5. As in Fig. 4, but showing the kinematics of two waves with moderate speeds (left: 2007 Dec. 7; right: 2009 Feb. 13). Note that the waves have nearly constant speeds.

2009 Feb. 13) in Fig. 5. These waves start off in the range of $v_{1}=250-350 \mathrm{~km} \mathrm{~s}^{-1}$ and subsequently propagate at a more or less constant speed, showing only slight overall deceleration $(\bar{a}$ is a few tens of $\mathrm{m} \mathrm{s}^{-2}, \delta=0.85$ ). With their superior cadence, these EUVI observations thus prove that there are really at least two kinds of EUV waves with very different kinematical characteristics - this finding is definitely not an artifact of the inferior cadence of EIT, in contrast to what has been suggested by Long et al. (2008).

There was one EUVI event that showed markedly different characteristics than the rest -2008 Dec. 8. It had a very low initial speed and showed an erratic kinematical behavior, with several episodes of acceleration and deceleration, as can be seen in Fig. 6. While there was an overall acceleration, neither the polynomial nor the power-law function provide a particularly good fit to the distances and speeds $\left(\chi_{\text {red }}^{2}>2\right)$, in stark contrast to the other EUVI waves that showed a smooth evolution. Zhukov et al. (2009) have reported this behavior and taken it as evidence in favor of the magnetic reconfiguration scenario.

The different event classes should of course show up as distinct groups in kinematical plots that show the relationship between initial speed and both mean acceleration and power-law index. Therefore we perform, for both EUVI $195 \AA$ and $171 \AA$ observations, the same kind of analysis that has been done for the EIT waves in Sect. 2. In Fig. 7 (top left), $v_{1,195}$ is plotted against $\bar{a}_{195}$. As in the case of EIT waves, three groups of events can be 


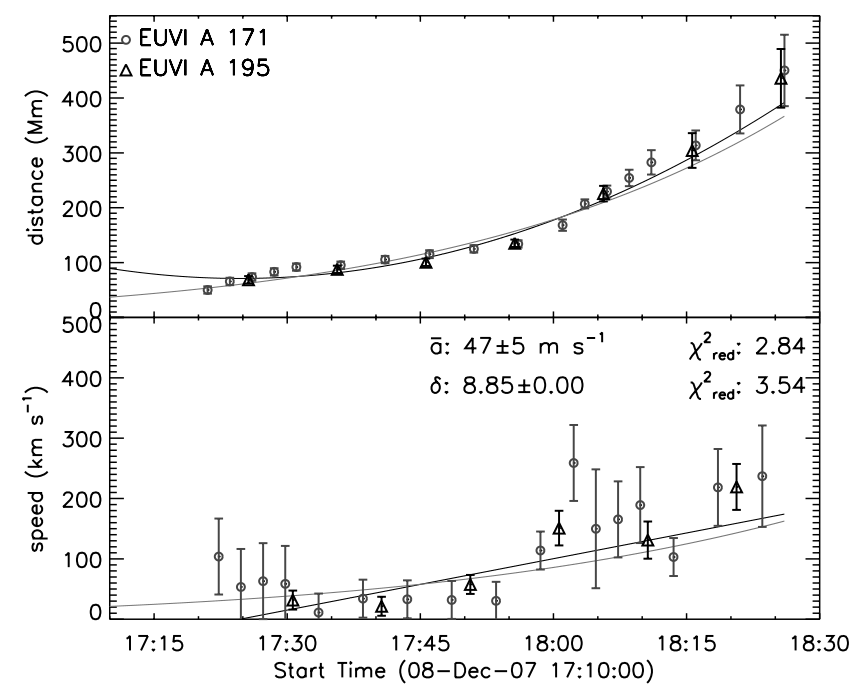

Fig. 6. As in Fig. 4, but showing the kinematics of the 2007 Dec. 8 wave event. Note the very low initial speed and the fact that neither the polynomial nor the power-law function provides a particularly good fit to the distances and speeds.

identified: decelerating fast waves $\left(v_{1,195}>370 \mathrm{~km} \mathrm{~s}^{-1}, \bar{a}_{195}<\right.$ $-100 \mathrm{~m} \mathrm{~s}^{-2}$ ) that show a clear correlation between initial speed and amount of deceleration, waves with moderate and nearly constant speeds $\left(v_{1,195}=220-370 \mathrm{~km} \mathrm{~s}^{-1},\left|\bar{a}_{195}\right|<75 \mathrm{~m} \mathrm{~s}^{-2}\right)$, and a single wave (2007 Dec. 8) that has a very low initial speed $\left(v_{1,195}=32 \mathrm{~km} \mathrm{~s}^{-1}\right)$ and shows some acceleration. $171 \AA \mathrm{ob}-$ servations (bottom left in Fig. 7) also show that initially fast waves decelerate strongly and slower waves have nearly constant speeds, although here it is more difficult to define event groups due to the low number of events. Note however that while speeds and accelerations as measured in the two EUVI channels agree quite closely for the slower waves, in the case of fast waves the $171 \AA$ data consistently yield higher speeds and also stronger deceleration. This is caused by the fact that the fast waves are not decelerating at a constant rate. Instead, the amount of deceleration decreases with time and distance, which means that the power-laws yield a better fit to the data than the 2 nd degree polynomials (cf. Fig. 4). Since the $171 \AA$ channel has a significantly higher cadence than the $195 \AA$ channel, it will sample the wave earlier in its evolution, which results in a higher initial speed (see also Long et al. 2008) and stronger deceleration.

Figure 7 (top right) shows the initial speed in the EUVI $195 \AA$ channel $v_{1,195}$ plotted against the power-law index $\delta_{195}$. Again, the results obtained from EIT can be verified: faster waves have a lower $\delta$, i.e. stronger deceleration, waves with moderate speeds have a $\delta$ close to unity, and the single slow wave is accelerating $(\delta>1)$. However, the two former event classes are not clearly separated but rather seem to form one single event group with an unbroken anticorrelation between initial speed and power-law index. The EUVI $171 \AA$ channel (bottom right in Fig. 7) basically reflects the same event groups. Here, the separation between fast decelerating and slower waves with nearly constant speeds is more pronounced than in $195 \AA$. Also note that in contrast to the mean accelerations, the power-law indices obtained from the two channels are very similar. This reflects the fact that the power-law better fits the kinematics of the decelerating waves.
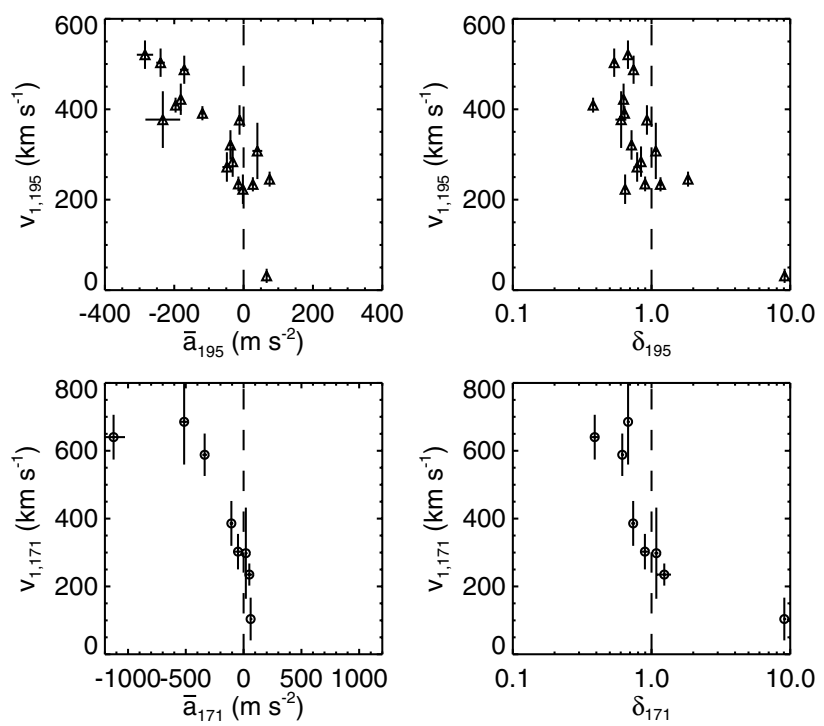

Fig. 7. Left: initial EUVI wave speed $v_{1}$ versus mean EUVI wave acceleration $\bar{a}$, obtained from $195 \AA$ images (top) and $171 \AA$ images (bottom), respectively. Right: initial EUVI wave speed $v_{1}$ versus power-law index $\delta$, derived from $195 \AA$ (top) and $171 \AA$ observations (bottom).

\section{Discussion}

\subsection{Kinematically distinct classes of coronal waves}

The distribution of EIT wave speeds, which shows several components, has suggested that there may be different classes of kinematically distinct coronal waves. This notion has been strengthened by considering the kinematical evolution of the waves. In particular, we have shown that the initial wave speed $v_{1}$ is an important factor for the waves' evolution. When $v_{1}$ is plotted as a function of the mean accelerations $\bar{a}$ or the powerlaw indices $\delta$ (which both parameterize the kinematical evolution), three distinct event groups can be identified. These groups are indicated in Fig. 8 for both the EIT waves of Thompson \& Myers (2009) (light green circles) and the EUVI waves (195 $\AA$ ) analyzed in this paper (dark green triangles). These groups represent event classes that can be characterized in the following manner:

- Class 1 events: Initially fast waves $\left(v_{1} \geq 325 \mathrm{~km} \mathrm{~s}^{-1}\right)$ showing deceleration $(\bar{a}<0, \delta<1)$, with faster waves decelerating more strongly.

- Class 2 events: Waves with initially moderate speeds $\left(v_{1} \approx\right.$ $170-320 \mathrm{~km} \mathrm{~s}^{-1}$ ) propagating with essentially constant velocities, or showing only slight acceleration or deceleration.

- Class 3 events: Initially slow waves $\left(v_{1} \leq 130 \mathrm{~km} \mathrm{~s}^{-1}\right)$ showing constant speeds or slight acceleration.

The means and standard deviations of the basic kinematical parameters of the three event classes are given in Table 2. This includes the initial and final speeds, $v_{1}$ and $v_{\text {last }}$, mean acceleration, $\bar{a}$, and power-law index, $\delta$, derived from the EIT as well as the EUVI $195 \AA$ and $171 \AA$ observations (no standard deviations are given for the EUVI class 3 since there was only one event in this category).

From both Fig. 8 and Table 2 it is evident that the results obtained from the high-cadence EUVI observations are consistent with the ones given by EIT. This validates the EIT results - the kinematical characteristics found have to be considered as real and not as being caused by insufficient sampling. While the separation between class 2 and class 3 events is very clear in Fig. 8, 
A. Warmuth and G. Mann: Kinematical evidence for physically different classes of large-scale coronal EUV waves
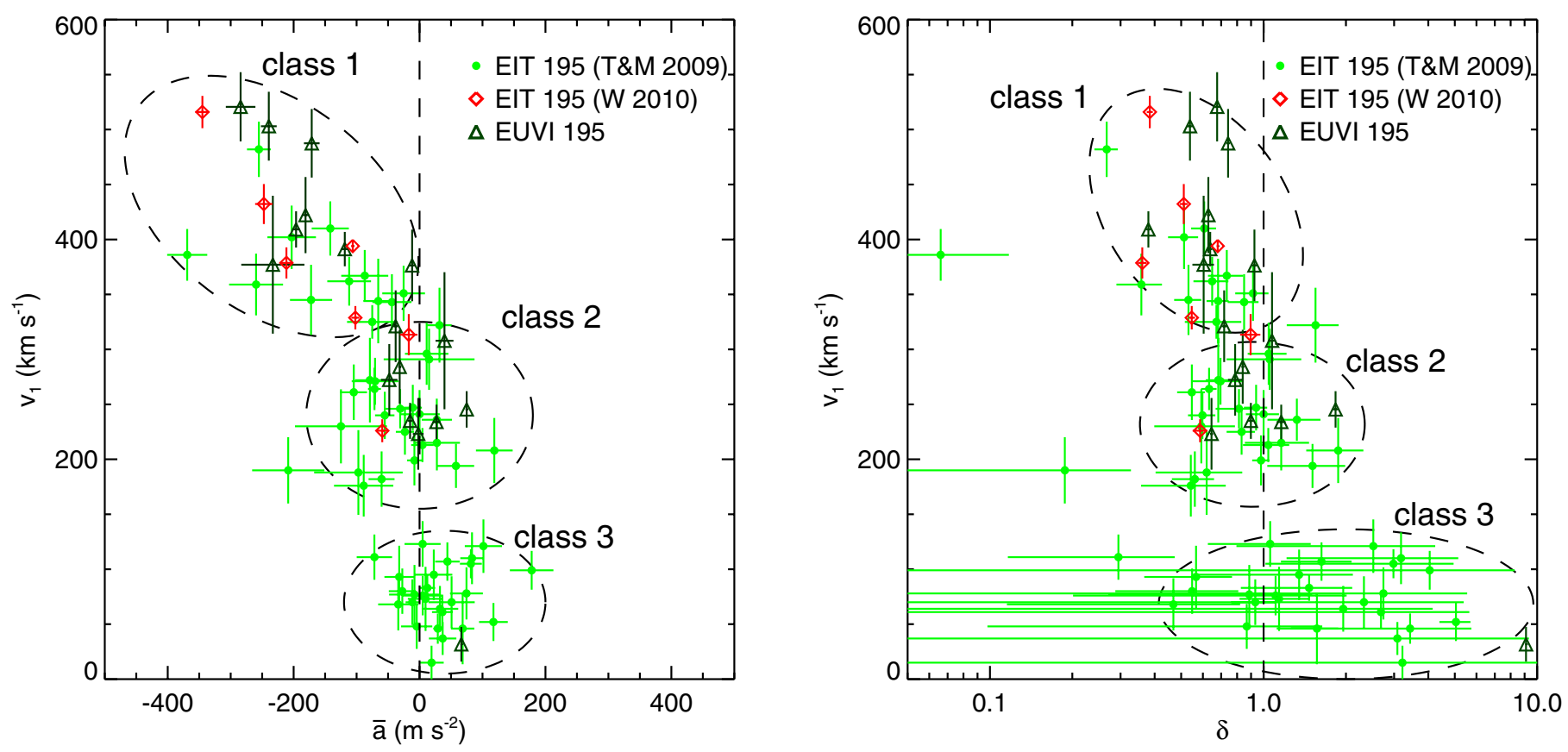

Fig. 8. Initial wave speed, $v_{1}$, versus mean wave acceleration, $\bar{a}(l e f t)$, and power-law index, $\delta(r i g h t)$. Filled light green circles represent the EIT waves measured by Thompson \& Myers (2009), red diamonds the EIT waves associated with the Moreton waves discussed by Warmuth (2010), and dark green triangles the EUVI waves presented in this paper. All parameters are derived from observations in the $195 \AA$ channel. The dashed ellipses denote the three proposed wave classes: class 1 events are initially fast and strongly decelerating waves, class 2 waves have moderate, nearly constant speeds, and class 3 events are very slow.

Table 2. Mean and standard deviation of the kinematical parameters for the three wave classes, as derived from EIT and EUVI $195 \AA$ A observations.

\begin{tabular}{|c|c|c|c|c|}
\hline Class & $\begin{array}{c}\left\langle v_{1}\right\rangle \\
\left(\mathrm{km} \mathrm{s}^{-1}\right)\end{array}$ & $\begin{array}{c}\left\langle v_{\text {last }}\right\rangle \\
\left(\mathrm{km} \mathrm{s}^{-1}\right)\end{array}$ & $\begin{array}{c}\langle\bar{a}\rangle \\
\left(\mathrm{m} \mathrm{s}^{-2}\right)\end{array}$ & $\overline{\langle\delta\rangle}$ \\
\hline \multicolumn{5}{|l|}{ EIT: } \\
\hline 1 & $373 \pm 43$ & $209 \pm 91$ & $-151 \pm 104$ & $0.57 \pm 0.24$ \\
\hline 2 & $235 \pm 39$ & $201 \pm 89$ & $-32 \pm 70$ & $0.9 \pm 0.39$ \\
\hline 3 & $77 \pm 27$ & $126 \pm 78$ & $31 \pm 54$ & $1.98 \pm 1.29$ \\
\hline \multicolumn{5}{|l|}{ EUVI 195 Å: } \\
\hline 1 & $436 \pm 59$ & $266 \pm 62$ & $-179 \pm 84$ & $0.64 \pm 0.16$ \\
\hline 2 & $265 \pm 37$ & $247 \pm 57$ & $1 \pm 43$ & $0.99 \pm 0.38$ \\
\hline 3 & 32 & 219 & 66 & 9.1 \\
\hline \multicolumn{5}{|l|}{ EUVI 171 A: } \\
\hline 1 & $638 \pm 49$ & $297 \pm 11$ & $-659 \pm 414$ & $0.56 \pm 0.15$ \\
\hline 2 & $305 \pm 62$ & $280 \pm 42$ & $-21 \pm 70$ & $0.99 \pm 0.22$ \\
\hline 3 & 104 & 237 & 61 & 9.06 \\
\hline
\end{tabular}

Notes. For details see main text.

the distinction between class 2 and class 1 is less pronounced, and there is some latitude in choosing a speed threshold (between 300 and $350 \mathrm{~km} \mathrm{~s}^{-1}$ ). A correlation between initial speed and acceleration is only evident for class 1 waves.

\subsection{The role of the final wave speeds}

After having studied the initial wave speeds, let us now consider the last measured speeds, i.e. the wave speeds at large distances, in more detail. In Fig. 9, $v_{\text {last }}$ is plotted as a function of the mean acceleration $\bar{a}$ and the power-law index $\delta$, as obtained from EIT $195 \AA$, EUVI $195 \AA$ and EUVI $171 \AA$ observations. With the exception of using the final speeds instead of the initial ones, these are the same kinematical plots as in Figs. 2, 3, 7 and 8. In contrast to these figures, Fig. 9 does not show distinct clusters of values that would suggest different event categories, and also no evident correlations. This suggests that the final speeds have no direct relation to the individual wave events.

While the EIT wave events show a very broad distribution of $v_{\text {last }}$, the final speeds of the EUVI waves are in a quite narrow range between 170 and $350 \mathrm{~km} \mathrm{~s}^{-1}$. Closer analysis shows that the lower EIT speeds are either associated with class 3 events, or are an artifact caused by coronal waves stopping at coronal hole boundaries (cf. the discussion in Sect. 2). Table 2 shows that on average both class 1 and class 2 events have similar final speeds $-\approx 200 \mathrm{~km} \mathrm{~s}^{-1}$ for the EIT waves and $\approx 260 \mathrm{~km} \mathrm{~s}^{-1}$ for the EUVI waves. Note that when events which show strong modification of their propagation due to interaction with coronal structures (see Sect. 2) are removed, the mean final EIT wave speeds for classes 1 and 2 are also close to $260 \mathrm{~km} \mathrm{~s}^{-1}$. This suggests that there is a link between class 1 and class 2 events.

In the following, we will discuss the significance of the various results on kinematics for the different wave classes and how they can be interpreted in terms of physics.

\subsection{Class 1 wave events - nonlinear waves/shocks?}

The most striking results for the fast waves were their ubiquitous deceleration and the correlation between initial speed and the amount of deceleration. This correlation can be easily explained by the fact that the fast waves all decelerate to comparable final speeds ( $v_{\text {last }} \approx 200-300 \mathrm{~km} \mathrm{~s}^{-1}$, as shown above in Sect. 4.2). An initially faster wave must decelerate more strongly in order to reach the same final speed. 

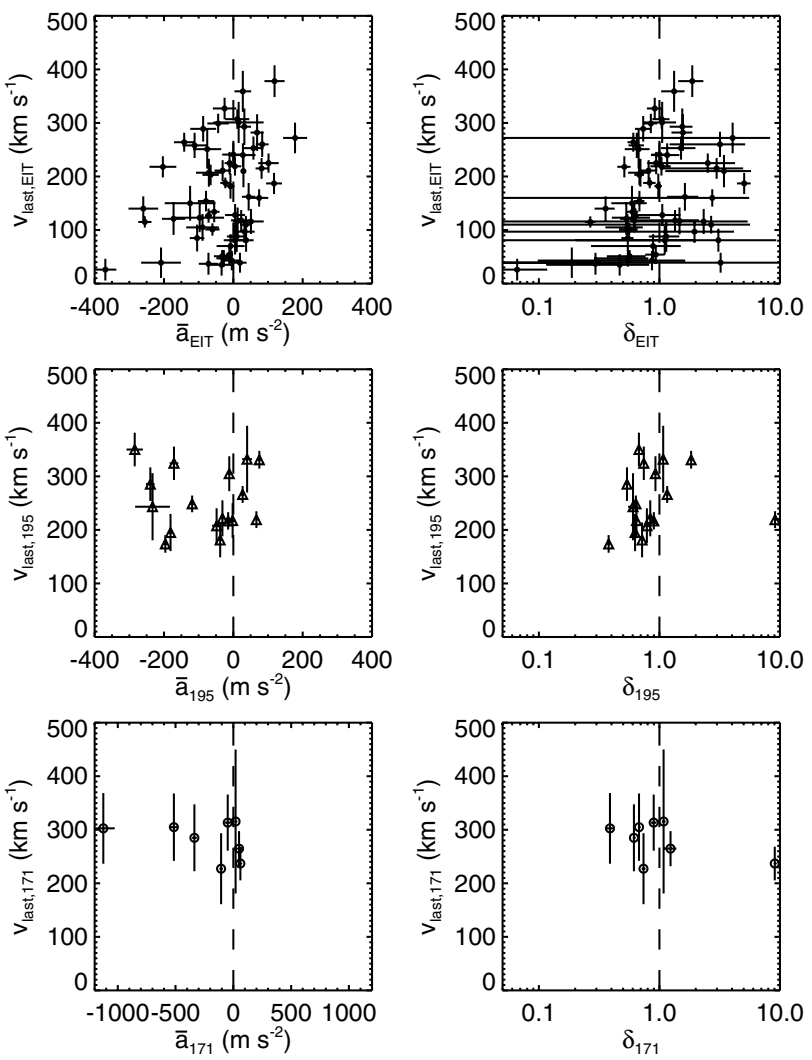

Fig. 9. Left: last measured wave speed $v_{\text {last }}$ versus mean wave acceleration $\bar{a}$, obtained from EIT $195 \AA$ (top) and EUVI $195 \AA$ (middle) and $171 \AA$ (bottom), respectively. Right: $v_{\text {last }}$ versus power-law index $\delta$, derived from EIT $195 \AA$ (top) and EUVI $195 \AA$ (middle) and $171 \AA$ (bottom).

Indeed all events studied so far featuring Moreton wave signatures show pronounced deceleration (see Warmuth 2010, for a study of 27 events), and are consistent with a single decelerating physical disturbance that generates wavefront signatures in the different spectral channels (cf. also Vršnak et al. 2006; Grechnev et al. 2008). The EUVI events considered here strongly support this conclusion, as has also been shown by other authors (e.g. Long et al. 2008; Veronig et al. 2008; Gopalswamy et al. 2009). The single decelerating perturbation is in contradiction to the scenario proposed by Chen et al. (2002) where Moreton and EIT signatures are interpreted in terms of two distinct disturbances. Also note that the average deceleration and power-law index of wave events with combined $\mathrm{H} \alpha$ and EIT observations $(\langle\bar{a}\rangle=$ $-284 \pm 162 \mathrm{~m} \mathrm{~s}^{-2}$ and $\langle\delta\rangle=0.63 \pm 0.1$ according to Warmuth et al. 2004a) is in good agreement with what was found for the class 1 waves (cf. Table 2).

For comparison, we have included in the kinematical plots in Fig. 8 the values for the EIT waves (those with more than two observed fronts) associated with the Moreton waves of Warmuth (2010), as well as for a further three events (1998 May 5, 2003 Nov. 3, and 2004 Nov. 10) for which no $\mathrm{H} \alpha$ data were available, but which had all the other characteristics of large-amplitude coronal waves: high initial speeds, sharp bright fronts, and associated metric type II bursts. The red diamonds in Fig. 8 show that five or six of these events fall into class 1 , while one or two fall into class 2. The two "slow events" are again a result of the low cadence of EIT, which has observed the EUV signatures of the corresponding coronal waves when they already had decelerated significantly. Considering concurrent data with higher cadences (H $\alpha$ and GOES-SXI) also shifts these events into class 1. Indeed all large-amplitude coronal waves shift to larger initial speeds and stronger decelerations when such high-cadence observations are included. This is just the result of the better sampling of the waves' initial phase where deceleration is most pronounced, as we have already discussed with respect to the EUVI $195 \AA$ and $171 \AA$ signatures in Sect. 3 .

In the classical wave model, coronal waves are considered as MHD waves that should propagate at their characteristic velocity. Since they are able to propagate perpendicularly to the predominantly radial magnetic field, they are considered as fast-mode waves. For perpendicular propagation, the fast-mode speed is the magnetosonic speed $v_{\mathrm{ms}}=\sqrt{c_{\mathrm{s}}^{2}+v_{\mathrm{A}}^{2}}$, with $c_{\mathrm{S}}$ as the sound speed and $v_{\mathrm{A}}$ as the Alfvén speed. Estimates for the magnetosonic speed in the low quiet corona range from some 200-300 $\mathrm{km} \mathrm{s}^{-1}$ (Mann et al. 1999, 2003; Warmuth \& Mann 2005) up to $300-600 \mathrm{~km} \mathrm{~s}^{-1}$ (Narukage et al. 2002). Thus most probably the class 1 waves are initially propagating faster than the characteristic speed. In case they are really MHD waves, they have to be at least partly shocked. This conclusion has been supported by soft X-ray observations (Narukage et al. 2002; Hudson et al. 2003), from which weak shock strengths have been deduced (with magnetosonic Mach numbers of $M_{\mathrm{ms}}=1.1-1.3$ ), while combined $\mathrm{H} \alpha /$ EIT observations have yielded somewhat stronger shocks $\left(M_{\mathrm{ms}}=2-3\right.$, see Warmuth \& Mann 2005).

A shock can be formed from a nonlinear large-amplitude fast-mode wave, a so-called simple wave (cf. Mann 1995; Vršnak \& Lulić 2000; Temmer et al. 2009). In such a wave, nonlinear terms can no longer be neglected, which leads to wave steepening and finally to the formation of a shock wave. The propagation speed of such a large-amplitude disturbance whether nonlinear wave or shock - is dependent on its amplitude, usually given in terms of the density compression factor, $X$. Due to the fact that the leading edge of the disturbance moves faster than its trailing edge, and due to the geometric expansion of the perturbation, its amplitude will drop during its propagation, leading to a deceleration. Finally, for small compressions $X \approx 1$ the large-amplitude disturbance will decay to an ordinary linear (i.e. small-amplitude) fast-mode wave, for which the phase velocity is no longer dependent on $X$.

This kinematical behavior is exactly what we find for the class 1 events. We therefore conclude that the most compelling physical interpretation for class 1 waves are large-amplitude nonlinear waves and/or shocks that subsequently decrease in amplitude and end up as linear waves. Another version of the shock scenario has been considered by Grechnev et al. (2008). They considered a spherical shock wave propagating in a medium with a radial density fall-off in terms of the Sedov solution. This model predicts power-laws for the kinematical curves of the disturbances. Note that we have actually found that power-laws provide a better fit of the kinematics than quadratic functions (see Sect. 3).

\subsection{Class 2 wave events - linear waves?}

Class 2 waves are propagating at nearly constant speeds, on average both their initial and final speeds are consistent with the final speeds of the class 1 events (some $200-300 \mathrm{~km} \mathrm{~s}^{-1}$; see Table 2). This implies two things: the speeds are not directly connected to the individual events, and there is a link between class 1 and class 2 waves. As discussed above, the speed range found is of 
the order of the coronal magnetosonic velocity. The kinematical characteristics of class 2 waves are thus fully consistent with linear fast-mode waves, for which the velocity does no longer depend on their amplitude. Their speed does not reflect their individual event characteristics, such as speed of a driver, but rather the ambient fast-mode speed.

We do not expect class 2 waves to have precisely constant speeds, since the local fast-mode speed is not constant, not even in the quiet corona. However we do not expect to see drastic changes, and recent high-cadence EUVI and SDO/AIA observations have confirmed that class 2 waves are consistent with this prediction (cf. Fig. 5; see also Veronig et al. 2010; Liu et al. 2010).

It has thus turned out that the wave/shock model nicely reproduces the characteristics of both class 1 and class 2 waves. Small-amplitude disturbances will propagate though the corona as linear MHD waves, while stronger perturbations will generate nonlinear waves (which may steepen to shocks) that will in turn transform to linear waves due to their expansion. Disturbances of medium strength would then only be initially nonlinear and then immediately decay to linear (or very weakly nonlinear) waves. Whether these events will be classified as class 1 or class 2 waves would then primarily depend on the temporal resolution of the observations. This is underlined by the fact that there is no clear separation between class 1 and class 2 events in the kinematical plots. Note that four of our class 2 events (those of April 2010) have recently been used in a study of homologous EUVI waves by Kienreich et al. (2011). Based on kinematics and wavefronts intensities, the authors find small perturbation amplitudes and derive magnetosonic Mach numbers close to unity. The fastest wave $\left(337 \mathrm{~km} \mathrm{~s}^{-1}\right)$ also had the highest Mach number $\left(M_{\mathrm{ms}} \sim 1.09\right)$. This is highly suggestive of the weakly nonlinear waves which we would expect to find near the boundary between class 1 and class 2 waves. We will discuss perturbation amplitudes in detail in a forthcoming paper, but preliminary results indicate that typically intensity amplitudes of class 2 waves are in the range of 5-10\% above background. This is in agreement with the results for the late phase of EIT waves obtained by Warmuth (2010) and implies that these disturbances are still consistent with linear waves.

\subsection{Class 3 wave events - magnetic reconfiguration?}

Class 3 waves, which are characterized by very low speeds, are difficult to understand in terms of the wave model because their measured velocities are actually smaller than all characteristic speeds in the corona. Even in the case of vanishing magnetic field strengths waves should travel at least with the sound speed $c_{\mathrm{s}}$, which for typical coronal temperatures of $T=1-1.5 \mathrm{MK}$ is $c_{\mathrm{s}}=151-185 \mathrm{~km} \mathrm{~s}^{-1}$. This implies that true MHD waves cannot propagate slower than some $150 \mathrm{~km} \mathrm{~s}^{-1}$ in the quiet corona. This cutoff can actually be seen as the gap in the kinematical plots (e.g. Fig. 8) that separates class 2 from class 3 events - there are no events with initial speeds between 130 and $170 \mathrm{~km} \mathrm{~s}^{-1}$. The same low-speed cutoff is seen for the final wave speeds as measured by EUVI (cf. Fig. 9). Events starting at even lower speeds are therefore most probably not MHD waves.

In addition, the 2007 Dec. 8 event has shown that class 3 waves can show very erratic kinematical behaviors - sudden decelerations and accelerations - that cannot be adequately fitted with a simple function. While a strong variation of density $N$ and magnetic field strength $B$ along the wave's propagation path could lead to such a behavior for MHD waves, no such strong variations are actually observed in the quiet corona (Warmuth \& Mann 2005).

All this implies that the wave/shock model, which has nicely reproduced the behavior of both class 1 and class 2 waves, is not able to explain the characteristics of the class 3 waves. We therefore have to consider the possibility that these events are generated by a different mechanism. The main alternative to the wave/shock model are different versions of a magnetic reconfiguration scenario, where an expanding CME interacts with the surrounding magnetic field and thus creates both stationary and moving bright fronts that are observed as coronal waves. The propagation speed of these fronts would be dependent on the kinematics of the expanding CME and could thus be well below the characteristic speeds of the corona. In addition, complex interaction with the surrounding magnetic fields could easily lead to erratic jumps in the fronts' propagation speed as observed in the 2007 Dec. 8 event (Zhukov et al. 2009).

An alternative model has been proposed by Wills-Davey et al. (2007), who suggested that coronal waves could be signatures of slow-mode solitons. In nonlinear waves, dispersive effects lead to the decay of the wave, whereas the nonlinearity leads to a wave steepening. A balance between dispersion and nonlinear steepening leads to the formation of a localized wave structure called soliton. Such large-scale wave phenomena can be described by MHD, which is however basically dispersionless. The inclusion of the Hall effect leads to the so-called Hall$M H D$, in which there are dispersive effects on the spatial scale of the ion inertial length $d_{\mathrm{i}}$ (see the discussion in Baumjohann \& Treumann 1996; and in Miteva \& Mann 2008). In Hall-MHD, the spatial scale of a soliton is of the order of few ion inertial lengths (see Miteva \& Mann 2008). $d_{\mathrm{i}}$ is defined by $d_{\mathrm{i}}=c / \omega_{\mathrm{pi}}$ with the velocity of light $c$ and the proton plasma frequency $\omega_{\mathrm{pi}}=\left(4 \pi e^{2} N_{\mathrm{p}} / m_{\mathrm{p}}\right)^{1 / 2}\left(e\right.$, elementary charge; $m_{\mathrm{p}}$, proton mass; $N_{\mathrm{p}}$, proton number density). In the fully ionized coronal plasma it is justified to assume $N_{\mathrm{p}} \approx N_{\mathrm{e}}$, with $N_{\mathrm{e}}$ as the electron number density. Coronal waves are mainly traveling in quiet coronal regions (cf. Warmuth \& Mann 2005), where the radial behavior of the electron number density can be described by a onefold Newkirk (1961) model, which agrees with a gravitationally stratified atmosphere with a temperature of $1.4 \times 10^{6} \mathrm{~K}$ (Mann et al. 1999). In the low corona, the Newkirk model yields electron densities of several $10^{8} \mathrm{~cm}^{-3}$. Adopting these values, we find ion inertial lengths on the order of $10 \mathrm{~m}$ in the solar corona, which some seven orders of magnitude smaller than the spatial width $l_{\mathrm{cw}}$ of these waves, which is in the range $l_{\mathrm{cw}}=20-200 \mathrm{Mm}$ (Warmuth 2010; Veronig et al. 2010). Due to this extreme ratio, dispersive effects are too weak to balance nonlinear effects such as wave steepening. In conclusion, coronal EUV waves cannot be solitons, and the most likely explanation for slow and/or irregularly propagating bright fronts is the magnetic reconfiguration scenario.

\section{Conclusions}

In science, we try to devise models that account for as many observational constraints as possible with the least assumptions. In other words, we search for unifying explanations. Taking an example out of the field of coronal waves, both the wave/shock and the hybrid model can account for the apparent speed discrepancy between Moreton and EIT waves. However, none of the proposed models for coronal waves is able to explain all the different - partly even contradictory - observational characteristics that have been reported. Instead, there is a tendency 
in the literature to strongly support one particular model as an explanation for coronal waves in general based on specific characteristics found in one or a few events. It is now very evident that the different physical models proposed were deduced from events that show fundamentally different kinematical characteristics. No consensus has been reached, so we have to consider the possibility that there is indeed no unifying model for coronal waves. This means we have to look for evidence for physically different classes of coronal waves.

Using the large sample of EIT waves of Thompson \& Myers (2009), we have found that there are three kinematically different classes of large-scale coronal EUV waves: initially fast and decelerating waves (class 1 ), waves with moderate and nearly constant speeds (class 2), and very slow and irregularly propagating waves (class 3). With high-cadence observations provided by STEREO/EUVI we have verified that these event classes are real and not an artifact caused by insufficient temporal sampling. We thus have to accept that large-scale globally propagating wavefronts in the solar corona really do show fundamentally different kinematical characteristics.

Accepting that there are different kinematical classes of coronal waves, and that very different kinematical behavior most likely implies that the various classes of events are generated by distinct physical processes, we can now try to determine which models are consistent with the different event classes. We found that the fast class 1 waves are consistent with a freely propagating shock formed from a nonlinear large-amplitude MHD wave. This model had already been strongly supported by observations of large-amplitude coronal waves showing $\mathrm{H} \alpha$ wavefronts and associated type II bursts (e.g. Klassen et al. 2000; Warmuth et al. 2004b; Grechnev et al. 2008; Balasubramaniam et al. 2010). The nearly constant speeds of the class 2 events suggests that these are linear (or only weakly nonlinear) waves propagating at (or very close to) the local fast-mode speed. Thus the combined wave/shock model is able to reproduce the characteristics of both class 1 and class 2 waves. In contrast to that, a non-wave mechanism has to be responsible for the slow and irregularly propagating class 3 events. The most likely candidate for that is some version of the magnetic reconfiguration scenario.

We do not claim that our association of the event classes with the different physical models holds for all events. While we maintain that the characteristics of the different event classes are best reproduced by specific models, we are aware that there may well be overlaps. For instance, one might envisage a magnetic reconfiguration event that - based on a particular magnetic topology - mimics either a class 1 or class 2 event. Conversely, a real MHD wave or shock may encounter strong inhomogeneities in ambient density and field strength, which would lead to an erratic propagation reminiscent of a class 3 event. Another possible scenario is that more than one of the proposed physical processes might be active in a single event. The possibility of an event having both a wave mode and an eruptive mode has been suggested both on theoretical grounds (e.g. Chen et al. 2002) as well as by observations (Zhukov \& Auchère 2004). In this respect, it is interesting to note that the first reported wave events using high-cadence SDO/AIA data did show multiple fronts with different speeds (Liu et al. 2010; Chen \& Wu 2011). This may imply that at least some coronal waves are much more complicated than our simple models would have suggested. These observational findings were only possible due to the extremely high cadence of AIA ( $\leq 20 \mathrm{~s})$, thus it is very important to systematically study coronal wave events with this powerful new instrument.
Kinematics are an essential characteristc of coronal waves, but it certainly is not the only important aspect. We therefore plan to study whether the kinematically defined event classes also show systematic differences in other respects, such as the perturbation profile and its evolution, or the association of the different wave classes with flares, CMEs and metric type II radio bursts. This will provide additional constraints for identifying the physical nature of the different event classes, and possible generation or launching mechanisms. Note that we have not discussed this latter aspect at all in the present work.

While there certainly remain many unsolved problems regarding coronal waves, we strongly feel that by identifying different event classes we have taken a significant step forward in our understanding of these phenomena. In differentiating between the event classes many of the apparent contradictions that have dominated the discussions in the past decade can be resolved. For future studies, we therefore urge the community to clearly identify which wave classes the events under consideration belong to. This can be easily achieved by determining the initial and final wave speeds as well as the "smoothness" of the kinematical curve.

Acknowledgements. This work was supported by DLR under grant No. 50 QL 0001. We thank the SOHO/EIT and STEREO/SECCHI teams for their open data policy.

\section{References}

Asai, A., Hara, H., Watanabe, T., et al. 2008, ApJ, 685, 622

Attrill, G. D. R., Harra, L. K., van Driel-Gesztelyi, L., \& Demoulin, P. 2007, ApJ, 656, L101

Balasubramaniam, K. S., Cliver, E. W., Pevtsov, A., et al. 2010, ApJ, 723, 587

Baumjohann, W., \& Treumann, R. A. 1996, Basic space plasma physics (London: Imperial College Press)

Chen, F., Ding, M. D., \& Chen, P. F. 2010, ApJ, 720, 1254

Chen, P. F., \& Wu, Y. 2011, ApJ, 732, L20

Chen, P. F., Wu, S. T., Shibata, K., \& Fang, C. 2002, ApJ, 572, L99

Chen, P. F., Fang, C., \& Shibata, K. 2005, ApJ, 622, 1202

Cohen, O., Attrill, G. D. R., Manchester, W. B., \& Wills-Davey, M. J. 2009, ApJ, 705,587

Dai, Y., Auchère, F., Vial, J.-C., Tang, Y. H., \& Zong, W. G. 2010, ApJ, 708, 913 Delaboudinière, J.-P., Artzner, G. E., Brunaud, J., et al. 1995, Sol. Phys., 162, 291

Delannée, C. 2000, ApJ, 545, 512

Delannée, C., \& Aulanier, G. 1999, Sol. Phys., 190, 107

Delannée, C., Török, T., Aulanier, G., \& Hochedez, J.-F. 2008, Sol. Phys., 247, 123

Gallagher, P. T., \& Long, D. M. 2010, Space Sci. Rev., 135

Gilbert, H. R., Daou, A. G., Young, D., Tripathi, D., \& Alexander, D. 2008, ApJ, 685,629

Gopalswamy, N., Yashiro, S., Temmer, M., et al. 2009, ApJ, 691, L123

Grechnev, V. V., Uralov, A. M., Slemzin, V. A., et al. 2008, Sol. Phys., 253, 263 Howard, R. A., Moses, J. D., Vourlidas, A., et al. 2008, Space Sci. Rev., 136, 67 Hudson, H. S., Khan, J. I., Lemen, J. R., Nitta, N. V., \& Uchida, Y. 2003, Sol. Phys., 212, 121

Kienreich, I. W., Temmer, M., \& Veronig, A. M. 2009, ApJ, 703, L118

Kienreich, I. W., Veronig, A. M., Muhr, N., et al. 2011, ApJ, 727, L43

Klassen, A., Aurass, H., Mann, G., \& Thompson, B. 2000, A\&AS, 141, 357

Liu, W., Nitta, N. V., Schrijver, C. J., Title, A. M., \& Tarbell, T. D. 2010, ApJ, 723, L53

Long, D. M., Gallagher, P. T., James McAteer, R. T. J., \& Bloomfield, D. Shaun. 2008, ApJ, 680, L81

Ma, S., Wills-Davey, M. J., Lin, J., et al. 2009, ApJ, 707, 503

Mann, G. 1995, J. Plasma Phys., 53, 109

Mann, G., Klassen, A., Estel, C., \& Thompson, B. J. 1999, in 8th SOHO Workshop: Plasma Dynamics and Diagnostics in the Solar Transition Region and Corona, ed. J.-C. Vial, \& B. Kaldeich-Schürmann (Noordwijk: ESA), ESA SP, 446, 477

Mann, G., Klassen, A., Aurass, H., \& Classen, H.-T. 2003, A\&A, 400, 329

Miteva, R., \& Mann, G. 2008, J. Plasma Phys., 74, 607

Moreton, G. E. 1960, AJ, 65, 494 
A. Warmuth and G. Mann: Kinematical evidence for physically different classes of large-scale coronal EUV waves

Moses, D., Clette, F., Delaboudinière, J.-P., et al. 1997, Sol. Phys., 175, 571

Muhr, N., Vršnak, B., Temmer, M., Veronig, A. M., \& Magdalenić, J. 2010, ApJ, 708,1639

Narukage, N., Hudson, H. S., Morimoto, T., et al. 2002, ApJ, 572, L109

Narukage, N., Ishii, T. T., Nagata, S., et al. 2008, ApJ, 684, L45

Newkirk, G. 1961, ApJ, 133, 983

Patsourakos, S., \& Vourlidas, A. 2009, ApJ, 700, L182

Patsourakos, S., Vourlidas, A., Wang, Y. M., Stenborg, G., \& Thernisien, A. 2009, Sol. Phys., 259, 49

Podladchikova, O., Vourlidas, A., Van der Linden, R. A. M., Wülser, J.-P., \& Patsourakos, S. 2010, ApJ, 709, 369

Temmer, M., Vršnak, B., Žic, T., \& Veronig, A. M. 2009, ApJ, 702, 1343

Thompson, B. J., \& Myers, D. C. 2009, ApJS, 183, 225

Thompson, B. J., Plunkett, S. P., Gurman, J. B., et al. 1998, Geophys. Res. Lett., 25,2465

Veronig, A. M., Temmer, M., \& Vršnak, B. 2008, ApJ, 681, L113

Veronig, A. M., Muhr, N., Kienreich, I. W., Temmer, M., \& Vršnak, B. 2010, ApJ, 716, L57

Vršnak, B. 2005, EOS Trans., 86, 112

Vršnak, B., \& Lulić, S. 2000, Sol. Phys., 196, 157
Vršnak, B., Warmuth, A., Brajša, R., \& Hanslmeier, A. 2002, A\&A, 394, 299

Vršnak, B., Warmuth, A., Temmer, M., et al. 2006, A\&A, 448, 739

Wang, H., Shen, C., \& Lin, J. 2009, ApJ, 700, 1716

Warmuth, A. 2007, in The High Energy Solar Corona: Waves, Eruptions, Particles, ed. K.-L. Klein, \& A. MacKinnon (Berlin Heidelberg: Springer Verlag), Lect. Notes Phys., 725, 107

Warmuth, A. 2010, Adv. Space Res., 45, 527

Warmuth, A., \& Mann, G. 2005, A\&A, 435, 1123

Warmuth, A., Vršnak, B., Aurass, H., \& Hanslmeier, A. 2001, ApJ, 560, L105

Warmuth, A., Vršnak, B., Magdalenić, J., Hanslmeier, A., \& Otruba, W. 2004a, A\&A, 418, 1101

Warmuth, A., Vršnak, B., Magdalenić, J., Hanslmeier, A., \& Otruba, W. 2004b, A\&A, 418, 1117

Warmuth, A., Mann, G., \& Aurass, H. 2005, ApJ, 626, L121

Wills-Davey, M. J., \& Attrill, G. D. R. 2009, Space Sci. Rev., 149, 325

Wills-Davey, M. J., DeForest, C. E., \& Stenflo, J. O. 2007, ApJ, 664, 556

Wu, S. T., Li, B., Wang, S., \& Zheng, H. 2005, J. Geophys. Res., 110, A11102

Wuelser, J., Lemen, J., Tarbell, T. D., et al. 2004, Proc. SPIE, 5171, 111

Zhukov, A. N., \& Auchère, F. 2004, A\&A, 427, 705

Zhukov, A. N., Rodriguez, L., \& de Patoul, J. 2009, Sol. Phys., 259, 73 